Pressure Gradient Effects On Two-Dimensional Plasma Expansion

S. Moon, R. F. Smith, J. Dunn, R. Keenan, J. Nilsen, J. R. Hunter, J. Filevich, J. J. Rocca, M. C. Marconi, V. N. Shlyaptsev

October 18, 2004

The 9th International Conference on X-ray Lasers Beijing, China

May 24, 2004 through May 28, 2004 
This document was prepared as an account of work sponsored by an agency of the United States Government. Neither the United States Government nor the University of California nor any of their employees, makes any warranty, express or implied, or assumes any legal liability or responsibility for the accuracy, completeness, or usefulness of any information, apparatus, product, or process disclosed, or represents that its use would not infringe privately owned rights. Reference herein to any specific commercial product, process, or service by trade name, trademark, manufacturer, or otherwise, does not necessarily constitute or imply its endorsement, recommendation, or favoring by the United States Government or the University of California. The views and opinions of authors expressed herein do not necessarily state or reflect those of the United States Government or the University of California, and shall not be used for advertising or product endorsement purposes. 


\title{
Pressure Gradient Effects On Two-Dimensional Plasma Expansion
}

\author{
S. Moon, R.F. Smith, J. Dunn, R. Keenan, J. Nilsen, J. R. Hunter \\ Lawrence Livermore National Laboratory, Livermore, CA 94551
}

\author{
J. Filevich, J.J. Rocca, M.C. Marconi \\ NSF ERC for Extreme Ultraviolet Science and Technology and \\ Department of Electrical and Computer Engineering, Colorado State \\ University, Fort Collins, Colorado 80523 \\ V.N. Shlyaptsev \\ University of California Davis-Livermore, Livermore, CA 94551
}

\begin{abstract}
Recent advances in interferometry has allowed for the characterization of the electron density expansion within a laser produced plasma to within $10 \mu \mathrm{m}$ of the target surface and over picosecond timescales. This technique employs the high brightness output of the transient gain Ni-like Pd collisional x-ray laser at $14.7 \mathrm{~nm}$ to construct an effective moving picture of the two-dimensional (2-D) expansion of the plasma. We present experimentally measured density profiles of an expanding Al plasma generated through laser irradiation in a $14 \mu \mathrm{m}$ line focus geometry. Significant lateral expansion was observed at all times as well as a pronounced on-axis electron density dip. Detailed modelling with a 2-D plasma physics code gives good agreement to experimental observations. Large pressure gradients associated with the tight focal spot conditions are calculated to dominate in shaping the plasma density profile.
\end{abstract}

\section{Introduction}

Understanding the underlying physical processes driving the expansion of laser produced plasmas is of considerable practical and fundamental interest. Combining well characterized experimental observables with detailed simulations can give a quantitative measure of conditions within the plasma. Interferometry is a powerful tool for accurately diagnosing the two-dimensional (2-D) evolution of dense laser-produced plasmas. The recently developed technique of picosecond x-ray laser interferometry combines the short wavelength of the $14.7 \mathrm{~nm}$ Ni-like Pd laser with diffraction grating interferometer (DGI) instrumentation to enable the direct measurement of the electron density profile within a laser-produced plasma to within $10 \mu \mathrm{m}$ of the target surface [1]. The picosecond duration of the x-ray laser probe pulse allows for an effective snapshot of the electron density profile while reducing the effects of plasma motion blurring at the ablation front. Also, the short wavelength, $\lambda$, and high brightness of the probe beam allows for 
characterization of large plasmas at high density with less deleterious effects from freefree absorption, $\propto \lambda^{3}(1-\exp [-h c / \lambda k T])$, and refraction $\left(\propto \lambda^{2}\right)$ [1]. This technique produces a series of high quality $2-\mathrm{D}$ density measurements providing unambiguous characterization of the time evolution in a fast evolving plasma for validation of existing 2-D hydrod ynamic codes.

\section{Experiment}

The electron density evolution of a laser-heated Al plasma is measured using a diffraction grating interferometer (DGI) [2] at different times, relative to the peak of a 600 ps plasma forming pulse. This gives an effective moving picture of how the plasma evolves in space and in time. The experimental results show pronounced two dimensional effects such as significant lateral transport and an on-axis density dip. We use 2-D hydrodynamic simulations in order to calculate the contributions of various physical mechanisms driving the plasma expansion. Density depressions have been observed before for high intensities laser drivers (pondermotive force [3]) and for lower intensities were the pumping pulse lasts for several nanoseconds (coronal x-ray ray heating [4]). For the plasma conditions explored here, simulations suggest that the on-axis density dip can be explained by rapid movement of material laterally due to the large pressure gradients set up by the tightly focused laser beam.

The Ni-like Pd $14.7 \mathrm{~nm}$ x-ray laser probe beam and the plasma to be studied were generated using three laser beams at $1054 \mathrm{~nm}$ wavelength from the COMET facility at LLNL [1]. Single pass saturated x-ray laser output of a few 10's of $\mu \mathrm{Js}$ was achieved with an optical pumping combination of a $600 \mathrm{ps}$ long pulse $\left(2 \mathrm{~J}, 2 \times 10^{11} \mathrm{~W} \mathrm{~cm}^{-2}\right)$ and a 13 ps $\left(5 \mathrm{~J}, 3.5 \times 10^{13} \mathrm{~W} \mathrm{~cm}^{-2}\right)$ main heating pulse. The $\mathrm{x}$-ray laser output was imaged and routed into a diffraction grating interferometer for plasma probing experiments. Recent temporal measurements [5] of the output of the Ni-like Pd x-ray laser have shown a $\sim 4 \pm$ $0.5 \mathrm{ps}$ output for the pumping conditions detailed above. For interferometry this sets the time period over which the electron density is sampled. For a detailed description of the interferometer instrumentation see ref [2]. A plasma heated by up to $3 \mathrm{~J}$ of energy in a 600 ps, $1054 \mathrm{~nm}$ pulse, corresponding to a maximum intensity of $1 \times 10^{12} \mathrm{~W} \mathrm{~cm}^{-2}$, was produced in one arm of the interferometer. A $3.2 \mathrm{~mm}$ long line focus with a $14 \mu \mathrm{m}$ focal width, was generated on a polished $1 \mathrm{~mm}$ long Al slab target using a combination of a cylindrical lens, $f=-200 \mathrm{~cm}$, and an off-axis paraboloid, $f=30 \mathrm{~cm}$. The temporal profile of the 600 ps plasma forming beam along with a cross section of the laser line focus are used as inputs into 2-D Lasnex simulations. The relative delay between the arrival of the $\mathrm{x}$-ray laser probe pulse, to the peak of the plasma forming beam was measured to within $100 \mathrm{ps}$ with a fast diode. The $\mathrm{x}$-ray laser could probe the plasma in the temporal range of $-1 \mathrm{~ns}$ to +2 ns relative to the peak of the 600 ps plasma forming pulse by adjusting a delay arm in the plasma laser beam. The line focus plasma was probed longitudinally by the $x$-ray laser, thereby minimizing uncertainties in the interpretation of the interferograms arising from plasma gradients along the probe path. The plasma was imaged with a magnification of $\sim 22$ by a $25 \mathrm{~cm}$ focal length spherical multilayer mirror and relayed to a thinned back-illuminated $1024 \times 1024$ CCD detector with $12.7 \times 12.7$ $\mu \mathrm{m}^{2}$ pixels. Using the $\mathrm{x}$-ray laser beam with no plasma present, high quality fringes, with visibility $V=\left(I_{\max }-I_{\min }\right) /\left(I_{\max }+I_{\min }\right)$ of $0.72 \pm 0.12$, were observed for a $700 \times 500 \mu \mathrm{m}^{2}$ $(\mathrm{H} \times \mathrm{V})$ region.

\section{Results}


Figure 1 shows a series of interferograms at different probing times relative to the peak of the plasma forming pulse. The electron density, $\mathrm{n}_{\mathrm{e}}$ in $\mathrm{cm}^{-3}$, is related to the measured fringe shifts as $N_{\text {fringe }}=6.68 \times 10^{-20} n_{e} L$, where $\mathrm{L}$ is the length $(\mathrm{cm})$ of the plasma being probed by the $14.7 \mathrm{~nm} x$-ray laser [6]. For the $1 \mathrm{~mm}$ plasma lengths described here, one fringe shift is equivalent to an electron density of $1.5 \times 10^{-20} \mathrm{~cm}^{3}$. An estimated uncertainty of $10-20 \%$ in the determination of the reference fringes results in an error of $1.5-3 \times 10^{19} \mathrm{~cm}^{-3}$ in the electron density. An additional phenomenon that can introduce errors in plasma probing experiments is the extent to which the probe beam is deflected by refraction in the plasma medium. Raytracing [7] has shown that this effect is negligible for the density gradients and plasma lengths considered here. The contribution of bound electrons to the refractive index within the plasma is calculated to be negligible for the plasma conditions studied here.

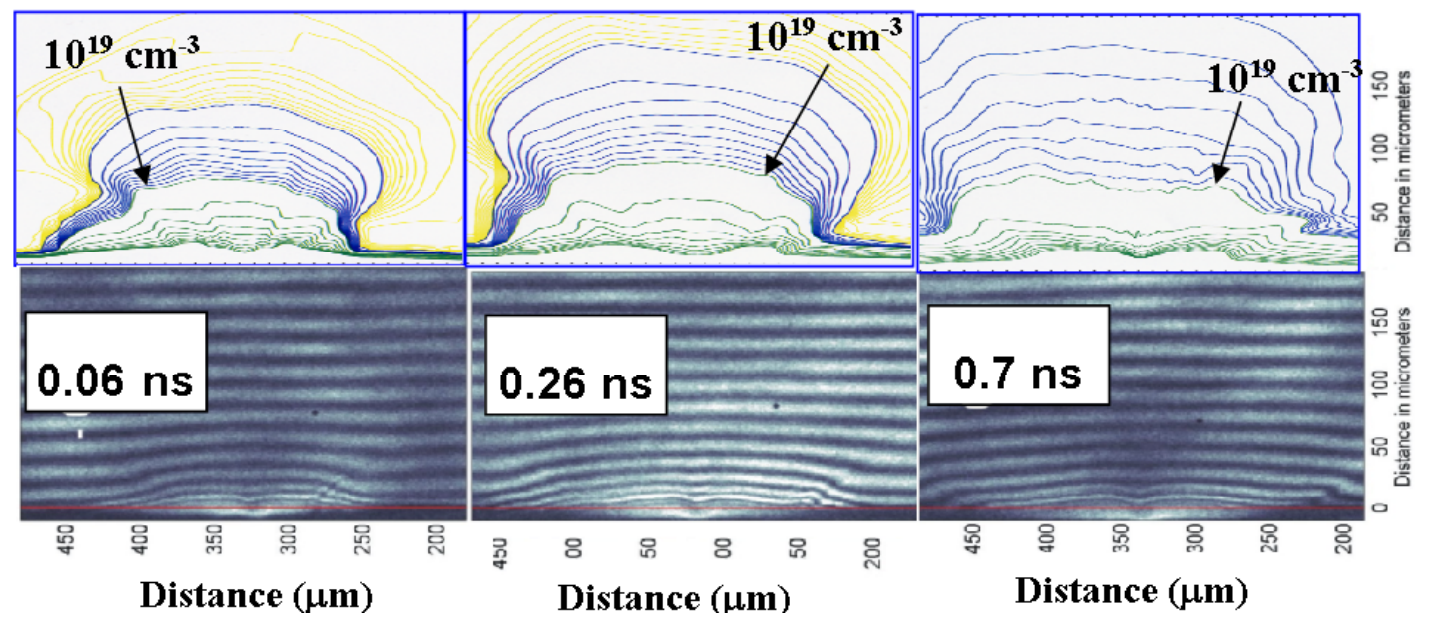

Fig 1. Experimentally obtained interferograms taken through longitudinal probing of an Al plasma at $0.06,0.26$ and $0.7 \mathrm{~ns}$ after the peak of the $600 \mathrm{ps}$ plasma forming pulse. Strong lateral expansion is observed from the original $14 \mu \mathrm{m}$ focal spot. Less than $20 \mu \mathrm{m}$ from the target surface there is a clear formation of a depression in the free-electron density. Shown in the three contour plots above are the calculated two-dimensional electron density profiles from the LASNEX plasma physics code. The extracted electron density contour map is shown in a logarithmic scale with each line connecting regions of equal density. Experimentally obtained spatial and temporal profiles for the irradiation beam were used as inputs into the simulations. The simulations give good agreement for the 2D plasma expansion and show the existence of an on-axis density dip.

Significant lateral expansion from the initial $14 \mu \mathrm{m}$ focal width is observed at all times. For all the interferograms the sideways expansion is symmetrical about the center of the focusing region. Within $10 \mu \mathrm{m}$ from the target surface there is a pronounced dip in the measured on-axis electron density profile. The low irradiation intensities $\left(\sim 10^{12} \mathrm{~W} / \mathrm{cm}^{2}\right)$ would suggest pondermotive forces could not explain such an effect [2]. Such a feature would be undetectable using traditional interferometric techniques which employed UV probe wavelengths due to severe refraction effects [7].

Also shown in Fig. 1 are contour plots of the electron density calculated from 2-D LASNEX [8] simulations. The experimentally determined temporal and spatial irradiation conditions were used as input into the code. Such modeling covers all phases of expansion leading to a plasma size much larger than the focal spot. With the 2-D simulations we have looked at several potential different driving mechanisms behind the plasma lateral expansion. Our preliminary findings suggest the dominant mechanism driving the evolution of the plasma is the strong pressure gradients set up by the tight line 
focus. By increasing the line focus to $50 \mu \mathrm{m}$ FWHM the on axis density dip is not observed.

Picosecond x-ray laser interferometry is a valuable technique in diagnosing plasma evolution within laser produced plasmas. The short sampling time of the probe beam reduces blurring effects and allows for probing to within $10 \mu \mathrm{m}$ of the target surface. In addition, the short wavelength probe minimizes effects associated with refraction and free-free absorption making this diagnostic well suited for studying large, fast evolving, dense plasmas. It has been shown that for the experimental conditions reported within this paper lateral expansion is a significant effect. Two-dimensional plasma physics codes are therefore necessary to model such experiments.

The support of Al Osterheld and Andy Hazi is greatly appreciated. The authors are pleased to acknowledge the technical contributions from Carl Bruns and Al Ellis. This work was performed under the auspices of the U.S. Dept. of Energy by the University of California Lawrence Livermore National Laboratory, through the Institute for Laser Science and Applications, under Contract No. W-7405-Eng-48 and by US Department of Energy Grant No. DE-FG03-98DP00208. This research was sponsored by the National Nuclear Security Administration under the Stewardship Science Academic Alliances program through DOE Research Grant \# DE-FG03-02NA00062. The CSU researchers also gratefully acknowledge the partial support of the NSF ERC Center for Extreme Ultraviolet Science and Technology, award number EEC-0310717 and the W.M. Keck Foundation.

\section{References}

[1] Smith R F, Dunn J, Nilsen J, Shlyaptsev V N, Moon S, Filevich J, Rocca J J, Marconi M C, Hunter J R, and Barbee T W, Jr., Phys. Rev. Lett., 89 (6), 0650041 (2002).

[2] Filevich J, Rocca J J, Marconi M C, Smith R F, Dunn J, Keenan R, Hunter J R, Moon S J, Nilsen J, Ng A, Shlyaptsev V N, "Picosecond resolution soft X-ray laser plasma interferometry", J. Appl. Phys., vol. 43, No. 19 (2003); Filevich J, Kanizay K, Marconi M C, Chilla J L A, and Rocca J J, "Dense plasma diagnostics with an amplitude-division soft-x-ray laser interferometer based on diffraction gratings" Opt. Lett. 25, 356=96358 (2000).

[3] Wilks S et al., Phys. Rev. Lett. 73, 2994 (1994).

[4] Filevich J, Rocca J J, Jankowska E, Hammarsten E C, Kanizay K, Marconi M C, Moon S J, Shlyaptsev V N, "Two-dimensional effects in laser-created plasmas measured with soft-x-ray laser interferometry", Phys Rev E, vol.67, no.5, pp.56409-1-6. (2003).

[5] Dunn J et al., submitted to Optics Letters (2004).

[6] Da Silva L B, Barbee T W, Jr., Cauble R, Celliers P, Ciarlo D, Libby S, London R A, Matthews D, Mrowka S, Moreno J C, Ress D, Trebes J E, Wan A S, and Weber F, Phys. Rev. Lett. 74, 3991-3994 (1995).

[7] Smith R F, Shlyaptsev V N, Dunn J, Nilsen J, Hunter J R, Rocca J J and Filevich J, Marconi M C, "Refraction Effects on X-ray and UV Interferometric Probing of Laser Produced Plasmas", JOSAB, vol.20, no.1, Jan. 2003, pp.254-9. (2002).

[8] Zimmerman G B and Kruer W L, Comments Plasma Phys. Controlled Fusion 2, 51-61 (1975). 